\title{
Baicalin alleviates TNBS-induced colitis by inhibiting PI3K/AKT pathway activation
}

\author{
LEI ZHU，HONG SHEN，PEI-QING GU，YA-JUN LIU，LU ZHANG and JIA-FEI CHENG \\ Department of Gastroenterology, The Affiliated Hospital of Nanjing University of Chinese Medicine, \\ Nanjing, Jiangsu 201129, P.R. China
}

Received July 23, 2018; Accepted November 6, 2019

DOI: $10.3892 /$ etm.2020.8718

\begin{abstract}
Inflammatory bowel diseases (IBDs) are chronic immunological disorders of the intestinal tract characterized by persistent inflammation. Baicalin, a type of flavonoid, has exhibited a wide range of pharmacological activities, including immunomodulation and anti-inflammation. However, little is known about the therapeutic role of baicalin in IBD. The aim of the present study was to ascertain whether baicalin could be a therapeutic drug of IBD and investigate its specific mechanisms. In the present study, the results revealed that baicalin not only significantly alleviated TNBS-induced colitis by reducing the release of IL-6, TNF- $\alpha$ and IL- $1 \beta$ and increasing the level of IL-10, but promoted the expression of tight-junction proteins ZO-1 and $\beta$-catenin, which may have been achieved by blockage of the PI3K/AKT signaling pathway. In vitro, the results demonstrated that baicalin clearly inhibited the release of TNF- $\alpha$, IL- 6 and IL- $1 \beta$ and promoted the expression of IL-10 in LPS-induced HT-29 cells, and significantly decreased LPS-induced HT-29 cell apoptosis by blockage of the PI3K/AKT signaling pathway. In conclusion, the present research revealed for the first time that baicalin acted as a therapeutic drug in IBD by suppression of the PI3K/AKT signaling pathway.
\end{abstract}

\section{Introduction}

Inflammatory bowel diseases (IBDs) are chronic, progressive immunologically-mediated diseases characterized by chronic inflammation of the gastrointestinal tract in genetically susceptible individuals exposed to environmental risk factors, encompassing ulcerative colitis and Crohn's disease (1-3). IBDs influence extensive populations and have been estimated

Correspondence to: Dr Hong Shen, Department of Gastroenterology, The Affiliated Hospital of Nanjing University of Chinese Medicine, 155 Hanzhong Road, Jianye, Nanjing, Jiangsu 201129, P.R. China

E-mail: shenhong2017@163.com

Key words: inflammatory bowel diseases, baicalin, TNBS, PI3K/AKT, LPS to affect 1.5 million Americans, 2.2 million people in Europe, and several hundreds of thousands more worldwide, and these numbers have been increasing with worsening of the environment and changing habitats $(3,4)$. Persistent, chronic inflammation of the gastrointestinal tract is assumed to underlie the causes of colitis-associated cancer (5), fibrosis (6), heart diseases (7) and even induces serious behavioral symptoms reflecting the effect of colitis on the central nerve system (8). The past decades have witnessed notable progress in understanding the development of IBDs, and various clinical trials aimed to interfere with IBDs have been performed, but the therapy of IBDs still remains intractable (9).

In the active state of IBDs, the intestinal epithelial barrier breaks down and cells infiltrate into the lamina propria. Cells from the innate immune system and adaptive immune system, consisting of neutrophils, monocytes, macrophages and T cells, release inflammatory cytokines $(2,10)$ and chemokines $(11,12)$, such as interleukin 6 (IL-6), tumor necrosis factor $\alpha$ (TNF- $\alpha$ ), interleukin-1 $\beta$ (IL-1 $\beta$ ), CXCL1 and CXCL9 (11). Persisting existence of inflammatory cytokines and chemokines exacerbate the apoptosis of epithelial cells leading to disruption of the epithelial barrier $(13,14)$. In accordance with the role of inflammatory cytokines in the progression of IBDs, a great amount of research and clinical trials have been conducted (15-17), but the efficacy of anti-TNF- $\alpha$ was not promising and was even accompanied with side effects, such as paradoxical psoriasis (18). These challenges need to be overcome.

Baicalin (5, 6, 7-trihydroxyflavone-7-b-D-glucuronide) is a major constituent isolated from the herb Huangqin, found in the root of Scutellaria baicalensis Georgi (19). Baicalin has been revealed to exhibit many pharmacological activities, such as anti-inflammation (20), antitumor (21), anti-apoptosis (22) and immunomodulation (23). It has been used to treat a series of diseases, including acute myocardial infarction (24) and renal ischemia-reperfusion injury (25). It has been revealed that baicalin could ameliorate isoproterenol-induced acute myocardial infarction through the p38/MAPK pathway (20), alleviate experimental colitis through blockage of the TLR4/NF- $\kappa$ B pathway (26), and attenuate symptoms of experimental autoimmune encephalomyelitis via activation of the SOCS3 pathway (27), however, the molecular mechanism of baicalin in treating IBDs still remains elusive.

In the present study, the underlying mechanisms of baicalin in the treatment of IBDs were investigated in both an animal 
model of 2,4,6-trinitrobenzene sulfonic acid (TNBS)-induced rat colitis and an lipopolysaccharide (LPS)-induced HT-29 cell inflammation model. In the present study, it was revealed that baicalin reduced the levels of IL- $1 \beta$, IL- 6 and TNF- $\alpha$ and increased the expression of IL-10, and ameliorated the apoptosis of intestinal mucosal epithelial cells and promoted the expression of tight-junction proteins in the PI3K/AKT-dependent pathway in vivo and in vitro. Therefore, for the first time, our results identified the mechanism by which baicalin affects the development of colitis through the PI3K/AKT-dependent signaling pathway.

\section{Materials and methods}

Chemicals and reagents. Baicalin (purity, 95\%; CAS registry no. 21967-41-9; molecular formula, C21H18O11; molecular weight, 446.36) and TNBS were obtained from Sigma-Aldrich; Merck KGaA. LPS Escherichia coli serotype 0111:B4 strain and LY294002 were purchased from InvivoGen, Inc. Rabbit anti-phospho-AKT (1:1,000; cat. no. 4691), anti-phospho-PI3K (1:1,000; cat. no. 17366), anti-total AKT (1:1,000; cat. no. 9272), anti-total PI3K (1:1,000; cat. no. 4255), anti-Bcl-2 (1:1,000; cat. no. 3498), anti-FasL (1:1,000; cat. no. 68405), anti-Bax (1:1,000; cat. no. 81740), anti-caspase-3 (1:1,000; cat. no. 14220), anti-caspase-9 (1:1,000; cat. no. 9508), anti-ZO-1 (1:1,000; cat. no. 13663), anti- $\beta$-Catenin (1:1,000; cat. no. 8480) and anti-GAPDH (1:1,000; cat. no. 5174) were purchased from Cell Signaling Technology, Inc.

Animals. A total of 30 Sprague-Dawley rats (aged 6-8 weeks; 15 male and 15 female; weighing 200-250 g) were purchased from the Model Animal Research Center of Nanjing University. All rats were maintained under environmentally-controlled conditions (ambient temperature, $22 \pm 2^{\circ} \mathrm{C}$; humidity $40 \%$ ) in a pathogen-free facility with a $12-\mathrm{h}$ light/dark cycle, and had access to water and food ad libitum.

All experimental procedures were performed in strict accordance with the Institutional Animal Care and Use Committee of Nanjing University of Chinese Medicine [approval no. SYXK(Su)2014-0004].

Induction of colitis. Colitis was induced by intracolonic administration of TNBS, as previously described (28). Briefly, rats that were fasted for $24 \mathrm{~h}$ with free access to water were lightly anesthetized with $350 \mathrm{mg} / \mathrm{kg}$ chloral hydrate, and a polyethylene catheter was inserted rectally until the splenic flexure (6-8 cm from the anus). Then, TNBS, dissolved in 50\% ethanol for a dose of $100 \mathrm{mg} / \mathrm{kg}, 0.25 \mathrm{ml}$ per rat, was administered through the catheter. After removing the catheter, the rats were held in a headfirst position for $60 \mathrm{sec}$ to prevent liquid outflow.

The rats were randomly divided into the following six groups: Control group (rats received $0.9 \%$ saline.); model group (TNBS-induced colitis without treatment, TNBS group); baicalin group (TNBS-induced colitis treated with baicalin, $100 \mathrm{mg} / \mathrm{kg} / \mathrm{d}$, per rat by gastric lavage); LY294002 group (TNBS-induced colitis treated with LY294002, $50 \mu \mathrm{g} / \mathrm{kg} / \mathrm{d}$, per rat via intravenous (i.v.) injection); IGF-1 group (TNBS-induced colitis treated with IGF-1, $1.5 \mu \mathrm{g} / \mathrm{kg} / \mathrm{d}$, per rat via intravenous (i.v.) injection); baicalin and IGF-1 co-admin- istration group (TNBS-induced colitis treated with baicalin, $100 \mathrm{mg} / \mathrm{kg} / \mathrm{d}$, per rat by gastric lavage, and IGF-1, $1.5 \mu \mathrm{g} / \mathrm{kg} / \mathrm{d}$, per rat via intravenous (i.v.) injection). The animals in each group were treated once a day for 14 days. In addition, the defecation times and shape of the feces in each group were observed. On the 15th day, the rats were sacrificed by cervical dislocation under anesthesia with a solution of ketamine $(100 \mathrm{mg} / \mathrm{kg}$, i.p.) and xylazine $(10 \mathrm{mg} / \mathrm{kg}$, i.p.), and the entire colon was removed from the cecum to the anus. The length and weight of the colon were measured. Then, colonic specimens were fixed immediately in a $10 \%(\mathrm{w} / \mathrm{v})$ neutral formalin solution or frozen in liquid nitrogen for further analyses.

TUNEL staining. TUNEL staining was performed to quantify cell apoptosis using a TUNEL Detection kit, according to the manufacturer's instructions. Briefly, sections were fixed with $4 \%$ paraformaldehyde at $37^{\circ} \mathrm{C}$ for $20 \mathrm{~min}$, immersed in the $3 \% \mathrm{H}_{2} \mathrm{O}_{2}$ at $37^{\circ} \mathrm{C}$ for $10 \mathrm{~min}$, and dipped into $0.1 \%$ TritonX-100 on ice for $2 \mathrm{~min}$. FITC-labeled dUTP $(50 \mu \mathrm{l})$ and terminal deoxynucleotidyltransferase from the kit was added to each section and then incubated at $37^{\circ} \mathrm{C}$ for $60 \mathrm{~min}$, followed by incubation with $50 \mu 1$ streptavidin-HRP from the kit at $37^{\circ} \mathrm{C}$ for $30 \mathrm{~min}$. After detection with the DAB kit for $10 \mathrm{~min}$ at $37^{\circ} \mathrm{C}$, sections were sealed with neutral gum. A total of six images of randomly selected visual fields were taken for each section with a fluorescence microscope (Olympus Corporation) at x400 magnification. TUNEL-positive cells in the ipsilateral hippocampus were observed and quantified with Image-Pro software (version 6.0.0.260). Brownish yellow particles in the nuclei of the cells in the hippocampal sections indicated TUNEL-positive, apoptotic cells. The apoptotic rate was calculated quantified with Image-Pro software (version 6.0.0.260; Media Cybernetics, Inc.).

Histology and immunohistochemistry. For hematoxylin and eosin (H\&E) staining, colons samples were fixed in $10 \%$ neutral formalin solution for $24 \mathrm{~h}$, dehydrated in increasing concentrations of ethanol, and embedded in paraffin. Thereafter, sections of tissue were cut at a thickness of $3 \mu \mathrm{m}$. For immunohistochemical staining, paraffin-embedded colon sections were deparaffinized, hydrated, and antigen-retrieved, and endogenous peroxidase activity was quenched by $3 \%$ $\mathrm{H}_{2} \mathrm{O}_{2}$ for $10 \mathrm{~min}$. Sections were then blocked with $5 \%$ bovine serum albumin for $20 \mathrm{~min}$, followed by incubation with anti-ZO-1 (cat. no. sc-33725; Santa Cruz Biotechnology, Inc.) and anti- $\beta$-catenin $(1: 1,000$; cat. no. 8480 ; Cell Signaling Technology, Inc.) overnight at $4^{\circ} \mathrm{C}$ and subsequently incubation with a secondary antibody $\operatorname{IgG}(1: 1,000$; cat. no. 3900; Cell Signaling Technology, Inc.) for $2 \mathrm{~h}$. Positive cells were visualized by adding DAB to the sections. Slides were viewed with a Nikon Eclipse 80i microscope equipped with a digital camera (DS-Ri1; Nikon Corporation).

Cell culture. The moderately differentiated human colon adenocarcinoma cell line HT-29 was obtained from ATCC (HTB-38TM). Cells were cultured in McCoy's 5A Medium supplemented with $10 \%$ fetal bovine serum (Invitrogen; Thermo Fisher Scientific, Inc.), $100 \mathrm{U} / \mathrm{ml}$ penicillin $\mathrm{G}$ potassium, and $100 \mu \mathrm{g} / \mathrm{ml}$ streptomycin at $37^{\circ} \mathrm{C}$ in a humidified atmosphere with $5 \% \mathrm{CO}_{2}$. Cells were seeded on a six-well 
culture plate and were allowed to grow to $70-80 \%$ confluence in complete medium containing $10 \% \mathrm{FBS}$ for $24 \mathrm{~h}$, and then the medium was changed to serum-free medium after washing twice with serum-free medium. LPS $(1 \mu \mathrm{g} / \mathrm{ml})$ was used to stimulate colonic adenocarcinoma HT-29 cells to induce inflammation.

Experimental cell groups. Growth-phase HT-29 cells were divided into six groups: Normal control group (blank); model control group (LPS); baicalin; LY294002; IGF-1 and baicalin + IGF-1 group. The blank group was incubated in McCoy's 5A medium without FBS for $24 \mathrm{~h}$; the medium was changed to fresh serum-free medium after washing twice with serum-free medium, and then LPS (1 mg/l) was added for $12 \mathrm{~h}$. The baicalin (316 $\mu \mathrm{g} / \mathrm{ml})$, LY29400 (0.158 $\mu \mathrm{g} / \mathrm{ml}), \mathrm{IGF} 1\left(4.8 \times 10^{-3} \mu \mathrm{g} / \mathrm{ml}\right)$ and baicalin $(316 \mu \mathrm{g} / \mathrm{ml})+\mathrm{IGF}-1\left(4.8 \times 10^{-3} \mu \mathrm{g} / \mathrm{ml}\right)$ groups were based on the stated drug interventions for $24 \mathrm{~h}$.

Western blotting. After surgery, the rats were sacrificed by cervical dislocation and the colons specimens were homogenized in radioimmunoprecipitation assay lysis buffer (Sunshine Biotechnology Co., Ltd.), then the supernatants were harvested by centrifugation at $6,036 \mathrm{x} \mathrm{g}$ for $10 \mathrm{~min}$ at $4^{\circ} \mathrm{C}$. HT-29 cells were stimulated with different concentrations of $\mathrm{H}_{2} \mathrm{O}_{2}(0,100,200,300,400,500$ and 1,000 $\mu \mathrm{M})$ for $24 \mathrm{~h}$, then washed twice with PBS, and protein was extracted by adding radioimmunoprecipitation assay lysis buffer on ice for $30 \mathrm{~min}$, and centrifuging at $6,036 \mathrm{x} \mathrm{g}$ for $10 \mathrm{~min}$ at $4^{\circ} \mathrm{C}$. Protein concentrations were determined using a bicinchoninic acid kit (Beyotime Institute of Biotechnology). Protein samples were homogenized with loading buffer and heated to $100^{\circ} \mathrm{C}$ for $5 \mathrm{~min}$, and $20 \mu \mathrm{g}$ of each sample was then resolved by $10 \%$ SDS-PAGE. Proteins were transferred to a polyvinylidene difluoride membrane (EMD Millipore) and were probed with primary and secondary anti-IgG (1:1,000; cat. no. 14708; Cell Signaling Technology, Inc.). Immunoreactivity was visualized using an enhanced chemiluminescence reaction kit (OriGene Technologies, Inc.) and quantified using ImageJ 5.0 software (National Institutes of Health).

Enzyme-linked immune absorbance assay (ELISA). The amount of TNF- $\alpha$, IL-6, IL-1 $\beta$ and IL-10 in the culture medium and in rat colon tissue extracts were measured with a commercial ELISA kit (R\&D Systems, Inc.) according to the manufacturer's instructions.

Annexin V/PI binding assay. Briefly, HT-29 cells were seeded on $100-\mathrm{mm}$ culture dishes to $70-80 \%$ confluence in complete medium containing $10 \%$ FBS for $24 \mathrm{~h}$, and then changed to serum-free medium after washing twice with serum-free medium. Cells were treated with $\mathrm{H}_{2} \mathrm{O}_{2}(0,100,200,300,400$, 500 and $1,000 \mu \mathrm{M}$ ) for $24 \mathrm{~h}$, then harvested and washed twice, once in PBS and once in binding buffer. An Annexin V-FITC Apoptosis Detection kit (eBioscience, Inc.) was used to detect the translocation of phosphatidylserine from inner membrane to the outer leaflet of the plasma membrane. Cells were resuspended in Binding Buffer and the concentration was adjusted to $10^{6} / \mathrm{ml}$. FITC-conjugated Annexin V (5 $\left.\mu \mathrm{l}\right)$ was added to $100 \mu \mathrm{l}$ of the cell suspension. The tubes were gently mixed and incubated for $15 \mathrm{~min}$ at room temperature in the dark.
The unconjugated Annexin $\mathrm{V}$ was removed by a wash using binding buffer, and then $5 \mu \mathrm{l}$ of PI was added to $200 \mu \mathrm{l}$ of the binding buffer. Flow cytometric analysis was conducted within $2 \mathrm{~h}$ (BD FACSCanto II; BD Biosciences), with the reagents stored between 2 and $8^{\circ} \mathrm{C}$. Immediately after use, the remaining reagents were returned to cold storage $\left(2-8^{\circ} \mathrm{C}\right)$.

Statistical analysis. All data examined are presented as the mean \pm SEM. Statistical analysis of the data was performed using SPSS software (17.0 for Windows, IBM Inc.). Comparisons between two groups were performed using Student's t-test. Comparisons among three or more groups were performed using one-way ANOVA, followed by the Tukey test. $\mathrm{P}<0.05$ was considered to indicate a statistically significant difference.

\section{Results}

Baicalin protects TNBS-induced colitis in rats via inhibition of PI3K/AKT pathway activation. Sprague-Dawley rats (weighing 200-250 g) were intracolonically administered TNBS to induce colitis, as previously described (28). To investigate the role of the PI3K/AKT pathway in the development of colitis, the PI3K/AKT pathway was inhibited or activated by the use of LY294002 or IGF-1. The results of western blotting revealed that LY294002 could markedly inhibit the activation of the PI3K/AKT pathway, and IGF-1 could activate the PI3K/AKT pathway by increasing the expression of phosphorylated (p)-PI3K and p-AKT (Fig. 1A). H\&E staining indicated that severe mucosal injury was observed in TNBS-induced rats, characterized by increased neutrophils, epithelial cell disruption, massive bowel edema and distorted architecture of crypts, but the PI3K inhibitor LY294002 could markedly ameliorate the morphological change and histological damage of colon similar to baicalin administration. Conversely, IGF-1 administration exacerbated TNBS-induced colitis manifested by histological staining. However, the accelerated colitis syndrome induced by the combination of TNBS and IGF-1 could be suppressed by baicalin (Fig. 1B).

TNBS-induced colitis is characterized by the breakdown of the intestinal epithelial barrier, infiltration of both the innate and adaptive immune cells into the lamina propria, and these immune cells synthesize and release a host of proinflammatory and anti-inflammatory cytokines, including IL-6, IL-1 $\beta$ and TNF- $\alpha$. Furthermore, the levels of inflammatory cytokines were detected in colitis tissues. As revealed in Fig. 1C, TNBS significantly increased the expression of IL-6, TNF- $\alpha$ and IL-1 $\beta$ and inhibited the expression of IL-10 in colon specimens, compared with the control group, however, inhibition of the PI3K/AKT pathway using LY294002 predominantly inhibited TNBS-induced inflammatory cytokine expression (IL-6, TNF- $\alpha$ and IL-1 $\beta$ ) and increased the expression of IL-10, compared with the TNBS group. Furthermore, the efficacy of LY294002 in suppressing inflammatory cytokine production was equivalent to baicalin administration in the dosage of $100 \mathrm{mg} / \mathrm{kg} / \mathrm{d}$. By contrast, IGF-1 efficiently increased the expression of IL- 6 , TNF- $\alpha$ and IL- $1 \beta$ and inhibited the expression of IL-10, compared with the TNBS group. However, this tendency could be rescued after baicalin administration, compared with the IGF-1 group (Fig. 1C). 
A

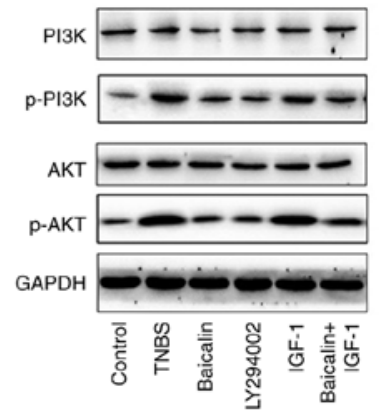

B
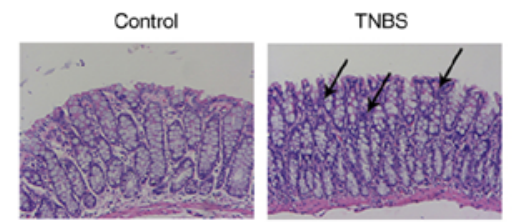

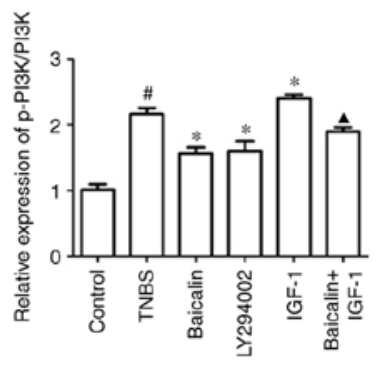

Baicalin
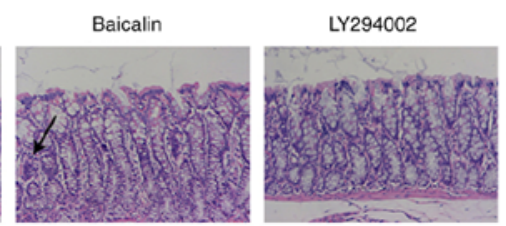
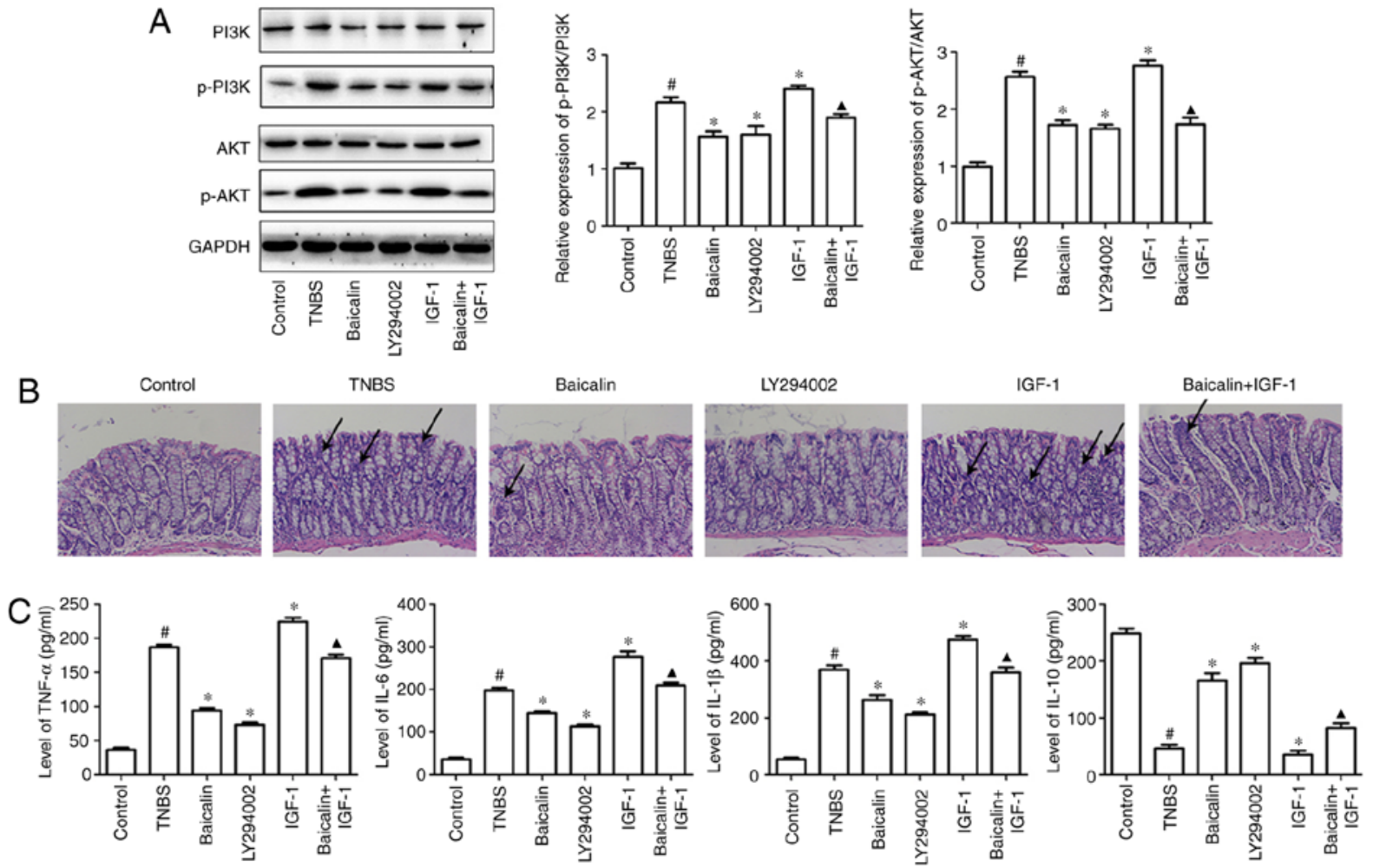

Figure 1. Baicalin protects TNBS-induced colitis in rats by inhibiting PI3K pathway activation. Colitis was induced by intracolonic administration of TNBS (100 mg/kg/d) for 14 days, then baicalin (100 mg/kg/d), LY294002 (50 $\mu \mathrm{g} / \mathrm{kg} / \mathrm{d})$, IGF-1 (1.5 $\mu \mathrm{g} / \mathrm{kg} / \mathrm{d})$ or IGF-1 along with baicalin were administrated for 14 days to investigate their role in TNBS-induced colitis. On the 15 th day, the rats were sacrificed and colons were homogenized, and $20 \mu \mathrm{g}$ of protein from lysates was analyzed for p-PI3K, p-AKT, PI3K and AKT expression (A) by western blotting. The other colonic specimens were fixed immediately in a $10 \%$ (w/v) neutral formalin solution for H\&E staining to observe the injury of the intestine. (B) The colons of the mice in the control group exhibited a normal structure without damage. However, in the TNBS model group, the colons exhibited mucosal ulcerations and inflammatory cell infiltration, however these alterations were attenuated to varying degrees by baicalin and LY294002. In addition, IGF significantly increased inflammatory cell infiltration, however the effect could be reversed by baicalin, as indicated by the arrows. (C) The supernatants of homogenized colons were also used to perform ELISA to detect IL-6, TNF- $\alpha$, IL-1 $\beta$ and IL-10 production. The data are representative of at least three independent experiments. Values are expressed as the mean \pm SEM of at least three independent experiments. ${ }^{\#} \mathrm{P}<0.05$ vs. the control group; ${ }^{*} \mathrm{P}<0.05$ vs. the TNBS group; ${ }^{\wedge} \mathrm{P}<0.05$ vs. the IGF-1 group.

These results demonstrated that the PI3K/AKT pathway was involved in baicalin-alleviated colitis response.

Baicalin ameliorates TNBS-induced intestinal mucosal cell apoptosis through blockage of the PI3K/AKT pathway. Apoptosis of the intestinal mucosal cell is a hallmark of TNBS-induced colitis, which disrupts intestinal mucosal integrity and barrier function, and leads to other changes associated with colitis (29). Excessive inflammation is considered to be a marker of apoptosis (30). In line with the results of inflammatory cytokine production in colon tissues, intestinal mucosal cell apoptosis was then investigated using TUNEL staining. As revealed in Fig. 2A, both baicalin and LY204002 significantly inhibited intestinal mucosal cell apoptosis, but IGF-1 induced cell apoptosis, which indicated the role of the PI3K/AKT pathway in colitis development. Moreover, when baicalin was administered in TNBS and IGF-1-treated rats, intestinal mucosal cell apoptosis could be inhibited by baicalin (Fig. 2A), which confirmed that baicalin exerted the suppression partly through inhibition of the PI3K/AKT pathway.

In order to ascertain the role of the PI3K/AKT pathway in baicalin-suppressed cell apoptosis, colon tissues lysates were used to detect the pro-apoptotic and anti-apoptotic proteins by western blotting. It was revealed that baicalin or LY294002 administration significantly suppressed TNBS-induced pro-apoptotic caspase-3, caspase-9, Bax and FasL expression, but increased the expression of Bcl-2, an anti-apoptotic protein, when compared with the TNBS group. In addition, increased pro-apoptotic protein caspase-9 expression was observed with IGF-1 treatment, however this effect could be reversed by baicalin treatment (Fig. 2B). Thus, baicalin suppressed TNBS-induced cell apoptosis through inhibition of the PI3K/AKT pathway.

Baicalin protects TNBS-induced colitis by increasing mucosal tight-junction proteins through inhibition of the PI3K/AKT pathway. Integrity of the intestinal epithelial cell (IEC) barrier plays an important role in maintaining mucosal immune homeostasis. Dysregulated IEC barrier function appears to trigger and perpetuate inflammation in IBD (31). To investigate the effects of baicalin and the PI3K/AKT pathway on the integrity of the IEC barrier, colon tissues underwent immunohistochemical staining for ZO-1 and $\beta$-catenin, the marker proteins of tight-junctions. The present results revealed that baicalin and LY294002 treatment could markedly increase ZO-1 and $\beta$-catenin expression compared to the TNBS-treated model group. In addition, the use of baicalin could alleviate IGF-1-induced decrease of tight-junction proteins (Fig. 3A), which indicated that baicalin exerted the protection of the IEC barrier via inhibition of PI3K/AKT pathway activation. 
A

Control

TNBS

Baicalin

LY294002

IGF-1

Baicalin+IGF-1
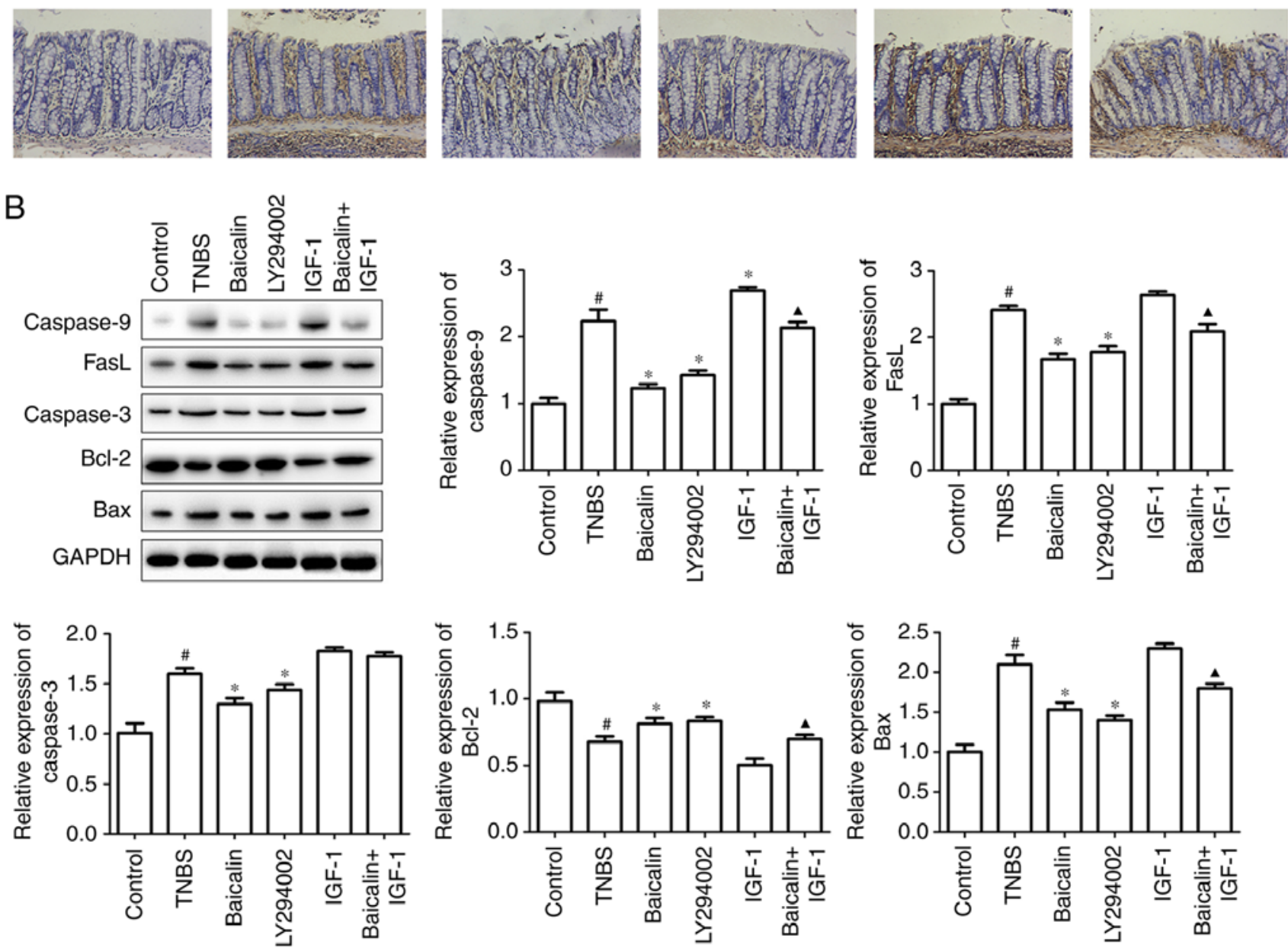

Figure 2. Baicalin ameliorates TNBS-induced intestinal mucosal cell apoptosis in PI3K/AKT-dependent manner. (A) The colon samples of rats underwent TUNEL staining to detect intestinal mucosal epithelial cell apoptosis in rats and slides were viewed with a Nikon Eclipse 80i microscope equipped with a digital camera (DS-Ri1; Nikon Corporation). (B) Western blot analyses revealed the pro-apoptotic proteins caspase-3, caspase-9, Bax, FasL and the anti-apoptotic protein Bcl-2 expression in colon lysates from the indicated group, and GAPDH was used as a loading control. ${ }^{~} \mathrm{P}<0.05$ vs. the control group; ${ }^{*} \mathrm{P}<0.05$ vs. the TNBS group; ${ }^{\wedge} \mathrm{P}<0.05$ vs. the IGF-1 group.
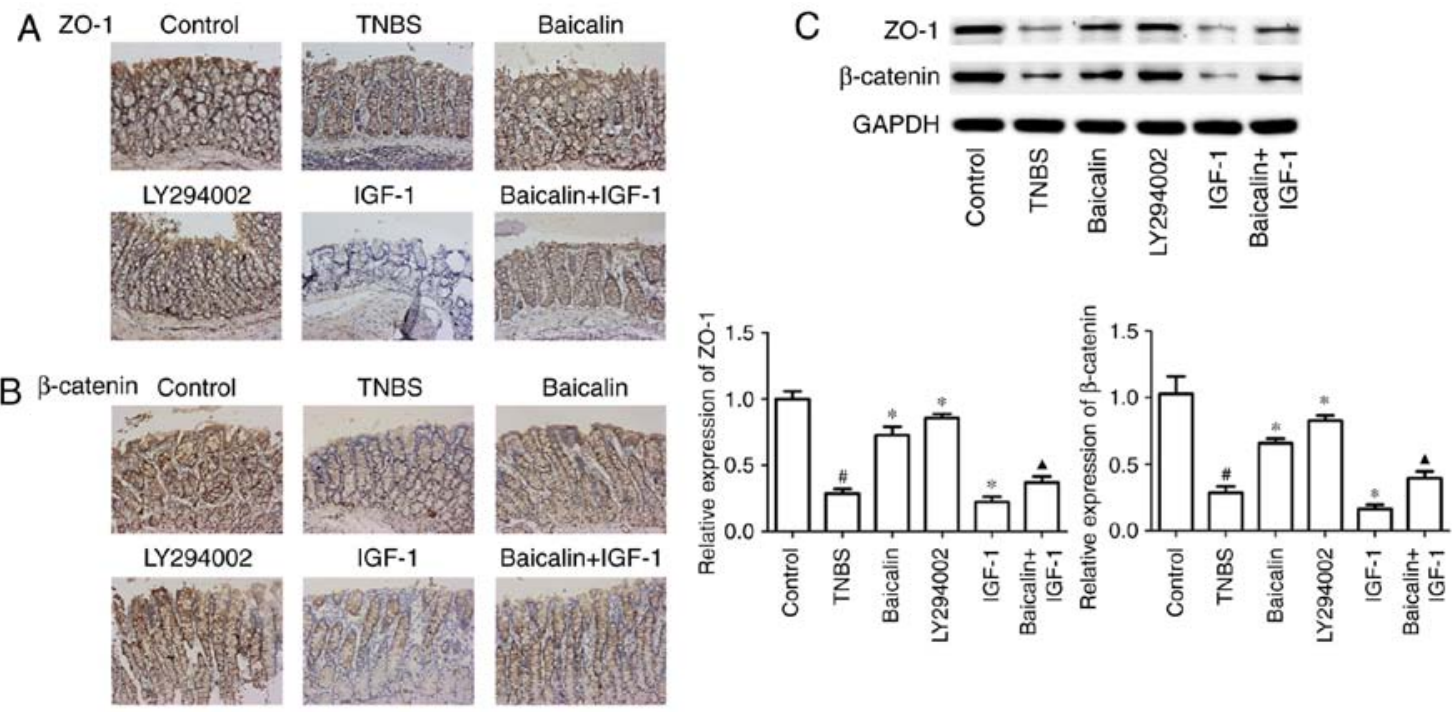

Figure 3. Baicalin protects TNBS-induced colitis by increasing mucosal tight-junction proteins in the PI3K/AKT-dependent pathway. (A and B) Immunohistochemistry was used to detect $\mathrm{ZO}-1$ and $\beta$-catenin expression in rat colons from of various groups. The sections were probed with the antibodies against ZO-1 (1:50) and $\beta$-catenin (1:100). (C) Western blot analyses revealed the ZO-1 and $\beta$-catenin expression in rat colons from each indicated group. Protein $(20 \mu \mathrm{g})$ from lysates was analyzed for ZO-1 and $\beta$-catenin, and GAPDH was used as an internal control. ${ }^{\sharp} \mathrm{P}<0.05$ vs. the control group; ${ }^{*} \mathrm{P}<0.05$ vs. the TNBS group; ${ }^{\wedge} \mathrm{P}<0.05$ vs. the IGF-1 group. 

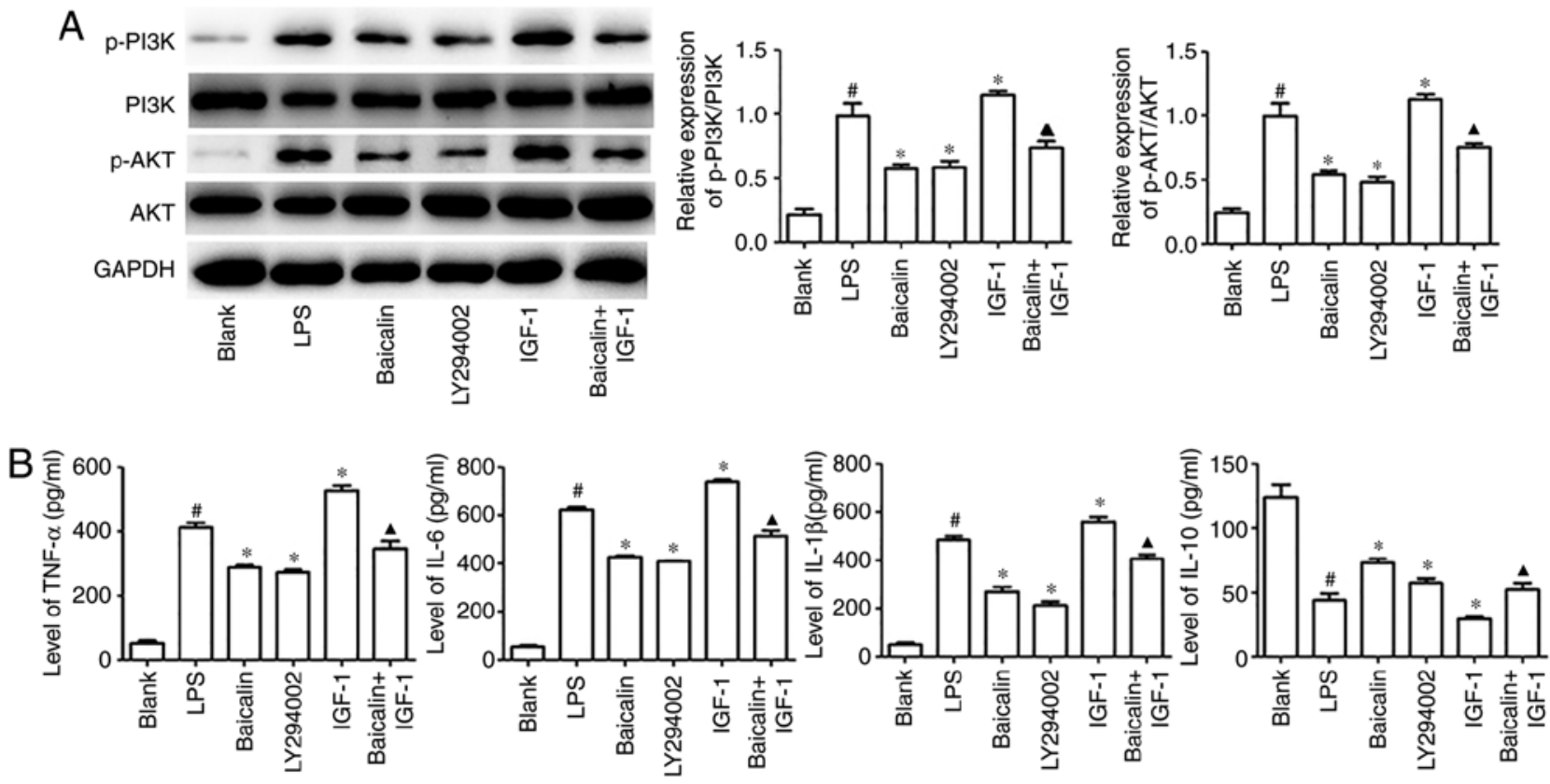

Figure 4. LPS-induced inflammation in HT-29 cells is suppressed by the use of baicalin. HT-29 cells were cultured for $24 \mathrm{~h}$ in complete medium, and then the medium was changed to serum-free medium after washing twice with serum-free medium. LPS (1 $\mu \mathrm{g} / \mathrm{ml})$ was used to stimulate HT-29 cells to induce inflammation. Then, baicalin $(100 \mathrm{ng} / \mathrm{ml})$, LY294002 $(50 \mu \mathrm{M})$ and IGF-1 $(0.2 \mu \mathrm{g} / \mathrm{ml})$ were used to investigate the role of baicalin and the PI3K pathway in LPS-induced inflammation. LY294002, IGF-1 and baicalin were used to treat cells for 30 min prior to LPS stimulation. Cell lysates were used to detect representative protein expression. (A) Western blot analyses showing the expression of phosphorylated PI3K and AKT, total PI3K, AKT and GAPDH were used as loading control. (B) HT-29 cells were treated and then the supernatants were collected to detect IL- 6 , TNF- $\alpha$, IL-1 $\beta$ and IL-10 concentrations by ELISA. Each column represents at least three independent experiments. Values are expressed as the mean $\pm \mathrm{SEM}$. ${ }^{\#} \mathrm{P}<0.05$ vs. the control group; ${ }^{*} \mathrm{P}<0.05$ vs. the LPS group; ${ }^{\wedge} \mathrm{P}<0.05$ vs. the IGF-1 group.

Furthermore, in accordance with the results of immunohistochemical staining, the results of western blotting revealed that baicalin and LY294002 increased ZO- 1 and $\beta$-catenin expression, compared with the TNBS group, moreover, baicalin also reversed IGF-1-induced reduction of ZO- 1 and $\beta$-catenin, compared with the TNBS group (Fig. 3C). These results indicated that baicalin protected IEC barrier integrity, which was a PI3K/AKT pathway inhibition process.

Baicalin suppresses LPS-induced inflammation in HT-29 cells. Epithelial cells are the critical first barrier for the intestine to defend itself from dangerous stimuli, and the most sensitive cells respond to hazardous substances. To study the role of baicalin in attenuating colitis, HT-29 cells were cultured, and LPS was used to induce epithelial cell injury. As revealed in Fig. 4A, when compared with the blank group, LPS induced significant activation of the PI3K/AKT pathway. However, when compared with the LPS group, baicalin markedly suppressed the levels of p-PI3K and p-AKT. Furthermore, IGF-1 was used to activate the pathway, and increased expression of p-PI3K and p-AKT was revealed compared to the LPS group. However, the influence of IGF-1 on LPS-stimulated epithelial cells could be reversed by baicalin (Fig. 4A), which indicated that baicalin was able to inhibit the PI3K/AKT pathway in HT-29 cells. Inflammatory cytokine production is the terminal result of pathway activation. Herein, baicalin significantly inhibited the LPS-induced IL-6, TNF- $\alpha$ and IL-1 $\beta$ production and promoted the expression of IL-10, and these effects were also observed with LY294002 treatment. In addition, baicalin treatment also reversed the IGF-1-induced increase of IL-6, TNF- $\alpha$ and IL-1 $\beta$ (Fig. 4B). These results indicated that baicalin ameliorated LPS-induced inflammation via the inhibition of PI3K/AKT activation in HT-29 cells.

Baicalin ameliorates apoptosis and tight-junction reduction by blockage of the PIJK/AKT pathway in HT-29 cells. To further demonstrate that baicalin could ameliorate cell death, flow cytometric analysis was employed to assess early apoptosis and late apoptosis. As presented in Fig. 5A, LPS significantly triggered colon cancer cell early apoptosis from 3.5 to $22.6 \%$ compared with the control group, whereas baicalin and LY294002 treatment decreased the early apoptosis rates from 22.6 to 10.5 and $8.33 \%$, respectively. Furthermore, baicalin markedly attenuated the early apoptosis rate to $12.6 \%$, which was significantly increased by IGF-1 treatment (26.2\%), as compared to the control group. Notably, the results also revealed that the various treatments did not cause any significant changes in late cell apoptosis, compared with the control group, indicating that the increase of apoptosis appeared to be possibly due to early apoptosis rather than late apoptosis. Western blotting results also revealed that when compared with the LPS group, baicalin and LY294002 suppressed pro-apoptotic caspase-3, caspase-9, Bax and FasL expression, but increased the expression level of Bcl-2 in HT-29 cells (Fig. 5B). In addition, the role of baicalin on tight-junction protein expression was explored in vitro, and the results revealed that baicalin and LY294002 reversed LPS-induced tight-junction protein decrease. These results indicated the vital role of baicalin on alleviating inflammation, apoptosis and tight-junction reduction, and this process involved PI3K/AKT pathway inhibition. 
A
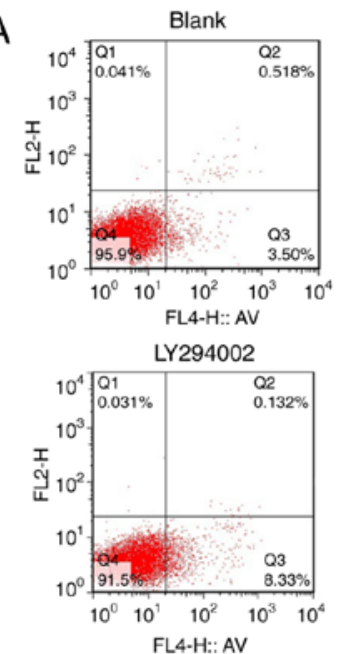
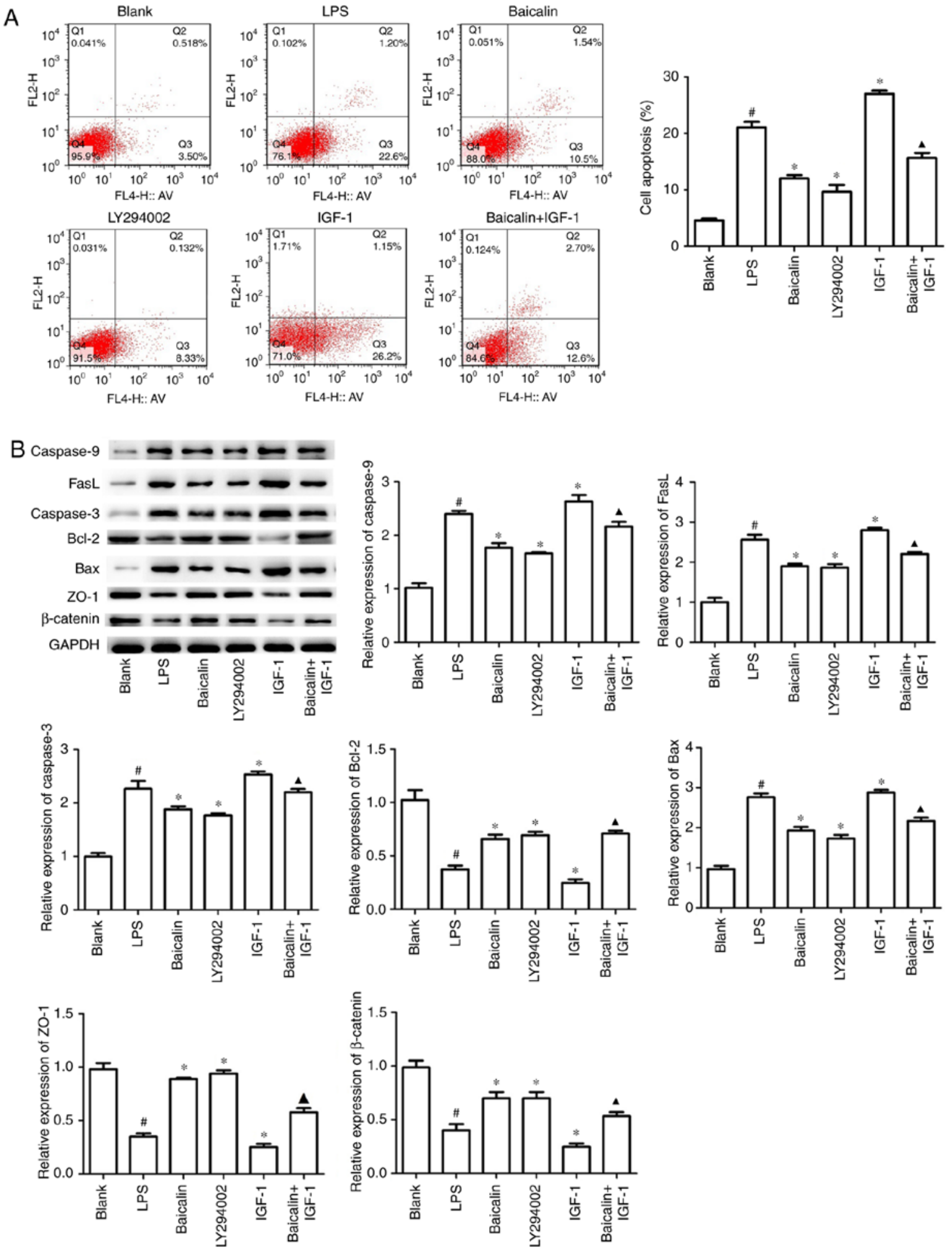

Figure 5. Baicalin inhibits the activation of the PI3K/AKT pathway in HT-29 cells to ameliorate apoptosis and tight-junction reduction. (A) HT-29 cells were harvested, and then cell apoptosis was detected using a commercial apoptosis kit and flow cytometry. (B) Western blotting was used to detect the protein expression of caspase-3, caspase-9, Bax, FasL, Bcl-2, ZO-1 and $\beta$-catenin levels using GAPDH as a loading control. "P<0.05 vs. the control group; ${ }^{*} \mathrm{P}<0.05$ vs. the LPS group; ${ }^{\wedge} \mathrm{P}<0.05$ vs. the IGF-1 group.

\section{Discussion}

Ulcerative colitis (UC) and Crohn's disease (CD) represent the two main forms of IBDs with clear-cut clinical and histological features. UC involves the rectum and colon, whereas
CD generally involves the colon and ileum. Both of them cause great pain and are costly to people and it has been estimated that 4.5 million people suffer from these diseases (32). Despite the recognized effect of IBD conventional therapies such as aminosalicylates, corticosteroids, and immunosuppressive 
agents, frequent adverse effects with these agents have been documented (33). Therefore, effective and safe treatments for IBDs are urgently required. Chinese herbs have been used to treat diseases for a long time. Nowadays, the use of their active ingredients is becoming an increasingly attractive approach to deal with various inflammatory disorders. Luan et al revealed that 50, 100 and $200 \mathrm{mg} / \mathrm{kg}$ of baicalin could attenuate rat myocardial ischemia-reperfusion injury through the AKT/NF-kB pathway (34). In an in vitro HT-29 model, 50, 100, 150 and $200 \mu \mathrm{M}$ of baicalin was used to study its role in colon cancer and the results revealed that baicalin could induce colon cancer cell apoptosis (35).

In the present study, the role of baicalin in treating TNBS-induced colitis in vitro and in vivo was investigated. The results demonstrated that baicalin alleviated the development of TNBS-induced colitis, reduced inflammation release, ameliorated intestinal mucosal cell apoptosis and increased the expression of tight-junction-associated proteins in vivo and in vitro. Notably, baicalin exerted a protective role in colitis via suppression of the PI3K/AKT pathway.

TNBS has long been used as a valuable model to explore the pathogenesis of IBDs. When TNBS is administered with ethanol, the mucosal barrier of the intestine can be broken by ethanol. TNBS is believed to haptenize colonic autologous or microbiota proteins rendering them immunogenic to the host immune system (34). The hapten-induced colitis has been characterized by dense infiltration of adaptive immune cells, predominantly $\mathrm{CD}^{+}{ }^{+} \mathrm{T}$ cells $(35)$, and innate immune cell-like macrophages (36). Based on inclination towards Th1 immune response which involves IL-12 and TNF- $\alpha$ as effector cytokines (35), baicalin was firstly investigated on inflammatory cytokine secretion. It was revealed that TNF- $\alpha$, a typical Th1-type cytokine, was significantly inhibited by the use of baicalin in rat colons, which indicated that baicalin can be an effective regulator of Th1-immune response. IL-6, produced by lamina propria $\mathrm{T}$ cells (37) and macrophages in the intestine, has been reported to be involved in the development of IBDs $(38,39)$. In the present study, it was reported that baicalin can also be an efficient inhibitor of IL-6 secretion.

Aberrant apoptosis of IECs is a hallmark of TNBS-induced colitis, which disrupts intestinal mucosal integrity and barrier function and leads to other changes associated with colitis $(40,41)$. TNF- $\alpha$, the main cytokine released in TNBS-induced colitis, is believed to play a vital role in the process of IEC apoptosis. Furthermore, anti-TNF- $\alpha$ therapies used for the treatment IBD patients were revealed to inhibit IEC apoptosis $(30,42)$. In accordance with the present results which revealed that baicalin inhibited TNF- $\alpha$ production, the inhibitory effect of baicalin on IEC apoptosis was also revealed, as observed by the fact that the expression of pro-apoptotic proteins such as caspase-3, caspase-9, Bax and FasL were suppressed in vivo and in vitro, and the anti-apoptotic molecule Bcl-2 was increased. Herein, HT-29 cells were used to evaluate the role of baicalin on inflammatory bowel diseases in vitro. HT-29 cells have been reported in numerous studies as targets for inflammatory bowel diseases in vitro (43-47). In future experiments, other cell lines will be selected to study the role of baicalin on inflammatory bowel diseases.

$\mathrm{PI} 3 \mathrm{~K}$ is an intracellular enzyme that catalyzes the phosphorylation of membrane inositol lipids and is involved in many biological processes, including cell growth, differentiation and survival (48). In the present study, it was reported that baicalin significantly suppressed the TNBS- or LPS-induced phosphorylation of PI3K and AKT, which indicated that baicalin exerted the protective effect in TNBS-induced colitis partly by inhibiting PI3K/AKT pathway activation. PI3K signaling was blocked by the PI3K specific inhibitor LY294002, and it was revealed that the effect of LY294002 on the inhibition of inflammatory cytokine production and intestinal epithelial cell apoptosis was equivalent to baicalin. Numerous studies on tumors have revealed the vital role of PI3K signaling in promoting tumor cell growth and resisting apoptosis. PI3K signaling even helps tumor cells obtain the ability to immortally grow $(49,50)$. However, in other cells except tumor cells, PI3K signaling appears to play an opposite role by promoting cell apoptosis to maintain a steady state (51). IECs are essential parts of the mucosal barrier. In order to maintain the balance and basic functions of the barrier, epithelial cells often enter the process of apoptosis upon various injury factors such as TNBS (52,53).

In conclusion, the present study demonstrated a novel mechanism in which baicalin attenuated TNBS-induced colitis via inhibition of PI3K/AKT activation, which was revealed by the decreased inflammatory cytokine levels, reduced IEC apoptosis and increased tight-junction proteins. These findings provide the first evidence of a direct link between traditional Chinese herbal ingredients and PI3K/AKT signaling in colitis, along with novel insight into the mechanisms of baicalin in treating colitis.

\section{Acknowledgements}

Not applicable.

\section{Funding}

The research was supported by The Natural Science Foundation of China (grant no. 81403344); the Special Scientific Research for Traditional Chinese Medicine of State Administration of Traditional Chinese Medicine of China (grant no. 201407001); the Jiangsu Provincial Special Program of Medical Science (grant no. BL2014100) and the National Chinese Medicine Clinical Research Base.

\section{Availability of data and materials}

All data generated or analyzed during the present study are included in this published article.

\section{Authors' contributions}

LeZ and HS designed the study and wrote the manuscript. PQG, YJL and LuZ performed the experiments. LuZ and JFC analyzed the data. HS supervised the work. All authors read and approved the final manuscript.

\section{Ethics approval and consent to participate}

All experimental procedures were performed in strict accordance with the Institutional Animal Care and Use Committee of Nanjing University of Chinese Medicine. 


\section{Patient consent for publication}

Not applicable.

\section{Competing interests}

The authors declare that they have no competing interests.

\section{References}

1. Bitton A, Vutcovici M, Patenaude V, Sewitch M, Suissa S and Brassard P: Epidemiology of inflammatory bowel disease in Quebec: Recent trends. Inflamm Bowel Dis 20: 1770-1776, 2014

2. Bashashati M, Rezaei N, Andrews CN, Chen CQ, Daryani NE Sharkey KA and Storr MA: Cytokines and irritable bowel syndrome: Where do we stand? Cytokine 57: 201-209, 2012.

3. Cosnes J, Gower-Rousseau C, Seksik P and Cortot A Epidemiology and natural history of inflammatory bowel diseases. Gastroenterology 140: 1785-1794, 2011.

4. Ananthakrishnan AN: Epidemiology and risk factors for IBD Nat Rev Gastroenterol Hepatol 12: 205-217, 2015.

5. Beaugerie L and Itzkowitz SH: Cancers complicating inflammatory bowel disease. N Engl J Med 372: 1441-1452, 2015.

6. Fichtner-Feigl S, Strober W, Geissler EK and Schlitt HJ: Cytokines mediating the induction of chronic colitis and colitis-associated fibrosis. Mucosal Immunol 1 (Suppl 1): S24-S27, 2008.

7. Rungoe C, Nyboe Andersen $\mathrm{N}$ and Jess T: Inflammatory bowel disease and risk of coronary heart disease. Trends Cardiovasc Med 25: 699-704, 2015.

8. Nyuyki KD and Pittman QJ: Toward a better understanding of the central consequences of intestinal inflammation. Ann NY Acad Sci 1351: 149-154, 2015.

9. Levesque BG, Sandborn WJ, Ruel J, Feagan BG, Sands BE and Colombel JF: Converging goals of treatment of inflammatory bowel disease from clinical trials and practice. Gastroenterology 148: 37-51 e1, 2015.

10. Fonseca-Camarillo $G$ and Yamamoto-Furusho JK Immunoregulatory pathways involved in inflammatory bowe disease. Inflamm Bowel Dis 21: 2188-2193, 2015.

11. Koelink PJ, Overbeek SA, Braber S, de Kruijf P, Folkerts G, Smit MJ and Kraneveld AD: Targeting chemokine receptors in chronic inflammatory diseases: An extensive review. Pharmacol Ther 133: 1-18, 2012.

12. Mitroulis I, Alexaki VI, Kourtzelis I, Ziogas A, Hajishengallis G and Chavakis T: Leukocyte integrins: Role in leukocyte recruitment and as therapeutic targets in inflammatory disease. Pharmacol Ther 147: 123-135, 2015.

13. Merga Y, Campbell BJ and Rhodes JM: Mucosal barrier, bacteria and inflammatory bowel disease: Possibilities for therapy. Dig Dis 32: 475-483, 2014.

14. Chen SJ, Liu XW, Liu JP, Yang XY and Lu FG: Ulcerative colitis as a polymicrobial infection characterized by sustained broken mucus barrier. World J Gastroenterol 20: 9468-9475, 2014.

15. Zhu Y, Mahon BD, Froicu M and Cantorna MT: Calcium and 1 alpha,25-dihydroxyvitamin D3 target the TNF-alpha pathway to suppress experimental inflammatory bowel disease. Eur J immunol 35: 217-224, 2005.

16. Danese S, Sans M, Scaldaferri F, Sgambato A, Rutella S, Cittadini A, Piqué JM, Panes J, Katz JA, Gasbarrini A and Fiocchi C: TNF-alpha blockade down-regulates the CD40/CD40L pathway in the mucosal microcirculation: A novel anti-inflammatory mechanism of infliximab in Crohn's disease. J Immunol 176: 2617-2624, 2006.

17. Condino G, Calabrese E, Zorzi F, Onali S, Lolli E, De Biasio F, Ascolani M, Pallone F and Biancone L: Anti-TNF-alpha treatments and obstructive symptoms in Crohn's disease: A prospective study. Dig Liver Dis 45: 258-262, 2013.

18. Pugliese D, Guidi L, Ferraro PM, Marzo M, Felice C, Celleno L, Landi R, Andrisani G, Pizzolante F, De Vitis I, et al: Paradoxical psoriasis in a large cohort of patients with inflammatory bowel disease receiving treatment with anti-TNF alpha: 5-year follow-up study. Aliment Pharmacol Ther 42: 880-888, 2015.

19. Ikemoto SK, Yoshida N, Yasumoto R, Wada S, Yamamoto K and Kishimoto T: Antitumor effects of Scutellariae radix and its components baicalein, baicalin, and wogonin on bladder cancer cell lines. Urology 55: 951-955, 2000.
20. Sun SJ, Wu XP, Song HL and Li GQ: Baicalin ameliorates isoproterenol-induced acute myocardial infarction through iNOS, inflammation, oxidative stress and P38MAPK pathway in rat. Int J Clin Exp Med 8: 22063-22072, 2015.

21. Li X, Zou K, Gou J, Du Q, Li D, He X and Li Z: Effect of baicalin-copper on the induction of apoptosis in human hepatoblastoma cancer HepG2 cells. Med Oncol 32: 72, 2015.

22. Guo X, Chi S, Cong X, Li H, Jiang Z, Cao R and Tian W: Baicalin protects sertoli cells from heat stress-induced apoptosis via activation of the Fas/FasL pathway and Hsp72 expression. Reprod Toxicol 57: 196-203, 2015.

23. Zou Y, Dai SX, Chi HG, Li T, He ZW, Wang J, Ye CG, Huang GL, Zhao B, Li WY, et al: Baicalin attenuates TNBS-induced colitis in rats by modulating the Th17/Treg paradigm. Arch Pharm Res 38: 1873-1887, 2015.

24. Liu X, Gu J, Fan Y, Shi H and Jiang M: Baicalin attenuates acute myocardial infarction of rats via mediating the mitogen-activated protein kinase pathway. Biol Pharm Bull 36: 988-994, 2013.

25. Lin M, Li L, Li L, Pokhrel G, Qi G, Rong R and Zhu T: The protective effect of baicalin against renal ischemia-reperfusion injury through inhibition of inflammation and apoptosis. BMC Complement Altern Med 14: 19, 2014.

26. Cui L, Feng L, Zhang ZH and Jia XB: The anti-inflammation effect of baicalin on experimental colitis through inhibiting TLR4/NF- $\kappa$ B pathway activation. Int Immunopharmacol 23: 294-303, 2014

27. Zhang Y, Li X, Ciric B, Ma CG, Gran B, Rostami A and Zhang GX: Therapeutic effect of baicalin on experimental autoimmune encephalomyelitis is mediated by SOCS3 regulatory pathway. Sci Rep 5: 17407, 2015.

28. Morris GP, Beck PL, Herridge MS, Depew WT, Szewczuk MR and Wallace JL: Hapten-induced model of chronic inflammation and ulceration in the rat colon. Gastroenterology 96: 795-803, 1989.

29. Siggers RH and Hackam DJ: The role of innate immune-stimulated epithelial apoptosis during gastrointestinal inflammatory diseases. Cell Mol Life Sci 68: 3623-3634, 2011.

30. Marini M, Bamias G, Rivera-Nieves J, Moskaluk CA, Hoang SB, Ross WG, Pizarro TT and Cominelli F: TNF-alpha neutralization ameliorates the severity of murine Crohn's-like ileitis by abrogation of intestinal epithelial cell apoptosis. Proc Natl Acad Sci USA 100: 8366-8371, 2003.

31. Cario E: Barrier-protective function of intestinal epithelial Toll-like receptor 2. Mucosal Immunol 1 (Suppl 1): S62-S66, 2008.

32. Kaplan GG: The global burden of IBD: From 2015 to 2025. Nat Rev Gastroenterol Hepatol 12: 720-727, 2015

33. Baumgart DC and Sandborn WJ: Inflammatory bowel disease: Clinical aspects and established and evolving therapies. Lancet 369: 1641-1657, 2007.

34. Wirtz S, Popp V, Kindermann M, Gerlach K, Weigmann B, Fichtner-Feigl S and Neurath MF: Chemically induced mouse models of intestinal inflammation. Nat Protoc 12: 1295-1309, 20017.

35. Randhawa PK, Singh K, Singh N and Jaggi AS: A review on chemical-induced inflammatory bowel disease models in rodents. Korean J Physiol Pharmacol 18: 279-288, 2014.

36. Wallace KL, Zheng LB, Kanazawa Y and Shih DQ: Immunopathology of inflammatory bowel disease. World J Gastroenterol 20: 6-21, 2014.

37. Atreya R, Mudter J, Finotto S, Müllberg J, Jostock T, Wirtz S, Schütz M, Bartsch B, Holtmann M, Becker C, et al: Blockade of interleukin 6 trans signaling suppresses T-cell resistance against apoptosis in chronic intestinal inflammation: Evidence in Crohn disease and experimental colitis in vivo. Nat Med 6: 583-588, 2000.

38. Atreya R and Neurath MF: Involvement of IL-6 in the pathogenesis of inflammatory bowel disease and colon cancer. Clin Rev Allergy Immunol 28: 187-196, 2005.

39. Atreya R and Neurath MF: New therapeutic strategies for treatment of inflammatory bowel disease. Mucosal Immunol 1: $175-182,2008$.

40. Qiu W, Wu B, Wang X, Buchanan ME, Regueiro MD, Hartman DJ, Schoen RE, Yu J and Zhang L: PUMA-mediated intestinal epithelial apoptosis contributes to ulcerative colitis in humans and mice. J Clin Invest 121: 1722-1732, 2011.

41. Lin W, Ma C, Su F, Jiang Y, Lai R, Zhang T, Sun K, Fan L, Cai Z, Li Z, et al: Raf kinase inhibitor protein mediates intestinal epithelial cell apoptosis and promotes IBDs in humans and mice. Gut 66: 597-610, 2017.

42. Zeissig S, Bojarski C, Buergel N, Mankertz J, Zeitz M, Fromm M and Schulzke JD: Downregulation of epithelial apoptosis and barrier repair in active Crohn's disease by tumour necrosis factor alpha antibody treatment. Gut 53: 1295-1302, 2004. 
43. Gu L, Ge Z, Wang Y, Shen M and Zhao P: Activating transcription factor 3 promotes intestinal epithelial cell apoptosis in Crohn's disease. Pathol Res Pract 214: 862-870, 2018.

44. Rodrigues RC, Pocheron AL, Cappelier JM, Tresse O and Haddad N: An adapted in vitro assay to assess Campylobacter jejuni interaction with intestinal epithelial cells: Taking into stimulation with TNFa. J Microbiol Methods 149: 67-72, 2018.

45. Gu L, Zhao J, Zhang S, Xu W, Ni R and Liu X: Runt-related transcription factor 2 (RUNX2) inhibits apoptosis of intestinal epithelial cells in Crohn's disease. Pathol Res Pract 214: 245-252, 2018.

46. Li M, Zhang S, Qiu Y, He Y, Chen B, Mao R, Cui Y, Zeng Z and Chen M: Upregulation of miR-665 promotes apoptosis and colitis in inflammatory bowel disease by repressing the endoplasmic reticulum stress components XBP1 and ORMDL3. Cell Death Dis 8: e2699, 2017.

47. Yan L, Wang L, Bai J, Miao X, Zeng W, Hua X, Ni R, Zhang D and Tang Q: Chromosome region maintenance-1 (CRM1) regulates apoptosis of intestinal epithelial cells via p27kip1 in Crohn's disease. Clin Res Hepatol Gastroenterol 41: 445-458, 2017.
48. Engelman JA, Luo J and Cantley LC: The evolution of phosphatidylinositol 3-kinases as regulators of growth and metabolism. Nat Rev Genet 7: 606-619, 2006.

49. Fresno Vara JA, Casado E, de Castro J, Cejas P, Belda-Iniesta C and González-Baròn M: PI3K/Akt signalling pathway and cancer. Cancer Treat Rev 30: 193-204, 2004.

50. Engelman JA: Targeting PI3K signalling in cancer: Opportunities, challenges and limitations. Nat Rev Cancer 9: 550-562, 2009.

51. Chen W, Liu Y, Xue G, Zhang L, Zhang L and Shao S: Diazoxide protects L6 skeletal myoblasts from $\mathrm{H} 2 \mathrm{O} 2$-induced apoptosis via the phosphatidylinositol-3 kinase/Akt pathway. Inflamm Res 65: 53-60, 2016.

52. Schulzke JD, Ploeger S, Amasheh M, Fromm A, Zeissig S, Troeger H, Richter J, Bojarski C, Schumann M and Fromm M: Epithelial tight junctions in intestinal inflammation. Ann NY Acad Sci 1165: 294-300, 2009.

53. Johansson ME: Mucus layers in inflammatory bowel disease. Inflamm Bowel Dis 20: 2124-2131, 2014. 